\title{
Rhynchophylline ameliorates myocardial ischemia/reperfusion injury through the modulation of mitochondrial mechanisms to mediate myocardial apoptosis
}

\author{
QIAO-JI QIN ${ }^{1}$, LI-QIANG CUI ${ }^{2}$, PENG LI $^{1}$, YONG-BIN WANG ${ }^{1}$, XUE-ZHI ZHANG ${ }^{1}$ and MING-LEI GUO ${ }^{1}$ \\ ${ }^{1}$ Emergency Department, The Affiliated Hospital of Qingdao University, Qingdao, Shandong 266003; \\ ${ }^{2}$ Department of Anesthesiology, Chengyang People's Hospital, Qingdao, Shandong 266109, P.R. China
}

Received December 28, 2018; Accepted January 18, 2019

DOI: $10.3892 / \mathrm{mmr} .2019 .9908$

\begin{abstract}
Rhynchophylline (RP), the primary active ingredient of Uncaria rhynchophylla, has an anti-hypertensive effect and protects against ischemia-induced neuronal damage. The present study aimed to examine the roles and mechanisms of RP in myocardial ischemia-reperfusion (MI/R) injury of rat cardiomyocytes. Cell viability, reactive oxygen species, mitochondrial membrane potential (MMP) and cell apoptosis were examined by a Cell Counting Kit- 8 assay and flow cytometry, respectively. An ELISA was performed to assess the expression of oxidative stress markers. Spectrophotometry was used to detect the degree of mitochondrial permeability transition pore (mPTP) openness. Western blotting and reverse transcription- quantitative polymerase chain reaction assays were used to evaluate the associated protein and mRNA expression, respectively. The present results demonstrated that RP increased the cell viability of MI/R-induced cardiomyocytes, and suppressed the MI/R-induced apoptosis of cardiomyocytes. Additionally, RP modulated the $\mathrm{Ca}^{2+}$ and MMP levels in MI/R-induced cardiomyocytes. Furthermore, RP decreased the oxidative stress and mPTP level of MI/R-induced cardiomyocytes. It was additionally observed that RP affected the apoptosis-associated protein expression and regulated the mitochondrial-associated gene expression in MI/R-induced cardiomyocytes. In conclusion, RP ameliorated MI/R injury through the modulation of mitochondrial mechanisms. The potential effects of RP on the protection of MI/R-induced apoptosis of cardiomyocytes suggest that RP may be an effective target for MI/R therapy.
\end{abstract}

Correspondence to: Dr Qiao-Ji Qin, Emergency Department, The Affiliated Hospital of Qingdao University, 16 Jiangsu Road, Qingdao, Shandong 266003, P.R. China

E-mail: qiaojiqin98av@163.com

Key words: Rhynchophylline, myocardial ischemia, reperfusion injury, reactive oxygen species, mitochondrial permeability transition pore, caspase-3/9, mitochondria

\section{Introduction}

An increasing trend has been observed in the incidence of coronary atherosclerotic heart disease, which is seen as one of the most common diseases that threatens human health (1). In 2008, the World Health Organization (WHO) released the 'Global Health Statistics Report' and reported that following malignant tumors, cardiovascular and cerebrovascular diseases have become the principal diseases leading to human mortality (2). Ischemic heart disease was reported to be the leading cause of mortality globally, resulting in $>8$ million deaths in 2013 (3). At present, the primary intervention in the therapy of AMI is to promptly open the myocardial infarction-associated artery and induce reperfusion by coronary artery bypass graft. However, it was identified that these treatments were accompanied by ischemia-reperfusion (I/R) injury, which impacts the effects of the treatment (4). I/R injury is the focus of cardiovascular research. The application of drug intervention following reperfusion may effectively reduce myocardial I/R (MI/R) injury (5-7). Therefore, the search for novel therapeutic agents to reduce the MI/R injury in the clinical setting is necessary.

Oxidative stress is a highly complex biological process in MI/R injury. During MI/R injury, a variety of adverse effects on the organism were observed, which further activated the neuroendocrine regulatory network through the hypothalamic-pituitary-adrenal axis and via sympathetic nervous system pathways $(8,9)$. Furthermore, the process of MI/R injury leads to abnormal cell proliferation, hypertrophy, differentiation and death, resulting in a series of pathophysiological effects $(10,11)$. The transmission and regulation of stress signals depends on the neuroendocrine network and cell-signaling network. Previous studies have demonstrated that in the pathological process of stress-induced cardiac disorders and cardiovascular diseases, MI/R injury, including cardiomyocyte necrosis and apoptosis, occurs (12-15). Overproduction of epinephrine and GCs in stress not only activates the protein kinase A system and other signaling pathways in cardiomyocytes (16); however, additionally enhances the expression of fibroblast-associated FAS and promotes the activation of caspase- 3 and -9 . These two phenomena may lead to an increased mitochondrial membrane potential (MMP) 
in cardiomyocytes. These processes further cause the release of mitochondrial cytochrome $c$ (cyto $c$ ), apoptosis-inducing factor (AIF) and second mitochondria-derived activator of caspase (Smac), leading to the apoptosis of cardiomyocytes (17-19). However, the accurate mechanisms of stress and mitochondria in MI/R injury remain unclear.

Uncaria rhynchophylla, a type of Rubiaceae plant, has been used for treating cerebral diseases (20). Uncaria rhynchophylla functions by lowering blood pressure, reversing cardiac hypertrophy, and reducing the force and frequency of cardiac muscle contraction (20). Rhynchophylline (RP), the primary active ingredient of Uncaria rhynchophylla, possesses an antihypertensive effect and protects against ischemia-induced neuronal damage $(21,22)$. Previous studies have demonstrated that RP is able to inhibit the proliferation of vascular smooth muscle cells and reduced angiotensin II-induced cardiomyocyte hypertrophy $(23,24)$. Other previous studies have additionally demonstrated that RP may be used in cerebral ischemia protection, calcium channel blocking, myocardial remodeling inhibition, anti-arrhythmia, anticonvulsant, and anti-inflammatory and analgesic therapy (25-27). However, little is known regarding the roles of RP in MI/R injury.

The present study aimed to investigate whether RP protects cardiomyocytes against MI/R injury. Furthermore, it was noteworthy to investigate the exact roles and mechanisms of RP and the mitochondrial mechanisms in MI/R injury.

\section{Materials and methods}

Establishment of cardiomyocyte oxygen-glucose deprivation/ reoxygenation $(O G D / R)$ injury to simulate $M I / R$ damage. The rat $\mathrm{H} 9 \mathrm{c} 2$ cardiomyocyte cell line was obtained from the Cell Bank of Chinese Academy of Sciences (Shanghai, China). To establish a model of $\mathrm{MI} / \mathrm{R}$ in vitro, the cardiomyocytes were first cultured in serum/glucose-free Dulbecco's modified Eagle's medium (DMEM; Gibco; Thermo Fisher Scientific, Inc., Waltham, MA, USA) and an anoxic environment (95\% $\mathrm{N}_{2}$ and $5 \% \mathrm{CO}_{2}$ ) at $37^{\circ} \mathrm{C}$ for $2 \mathrm{~h}$. The gas mixture of $95 \% \mathrm{~N}_{2}$ and $5 \% \mathrm{CO}_{2}$ was replaced with air at a flow velocity of $2 \mathrm{1} / \mathrm{min}$. Subsequent to the hypoxia process, the cardiomyocytes were transferred into fresh medium (DMEM) and maintained in an oxygen-rich incubator $\left(37^{\circ} \mathrm{C} ; 95 \% \mathrm{O}_{2}\right.$ and $\left.5 \% \mathrm{CO}_{2}\right)$. Following incubation for $1 \mathrm{~h}$, the MI/R cell model was harvested.

Grouping. Five treatment groups were prepared, which were the control group (cardiomyocytes with no treatment), MI/R group (cardiomyocytes treated with OGD/R injury), $20 \mu \mathrm{M}$ $\mathrm{RP}+\mathrm{MI} / \mathrm{R}$ group (cardiomyocytes treated with OGD/R injury, and subsequently treated with $20 \mu \mathrm{M} \mathrm{RP}$ at $37^{\circ} \mathrm{C}$ for $1 \mathrm{~h}$ ), $40 \mu \mathrm{M}$ RP+MI/R group (cardiomyocytes treated with OGD/R injury, and subsequently treated with $40 \mu \mathrm{M} \mathrm{RP}$ at $37^{\circ} \mathrm{C}$ for $1 \mathrm{~h}$ ) and $80 \mu \mathrm{M} \mathrm{RP}+\mathrm{MI} / \mathrm{R}$ group (cardiomyocytes treated with OGD/R injury, and subsequently treated with $80 \mu \mathrm{M}$ $\mathrm{RP}$ at $37^{\circ} \mathrm{C}$ for $\left.1 \mathrm{~h}\right)$. RP was purchased from Beijing Solarbio Science \& Technology Co., Ltd. (Purity $>98 \%$; Beijing, China).

Cell viability analysis. A Cell Counting Kit-8 (CCK-8; Beyotime Institute of Biotechnology, Haimen, China) was performed to assess the cell viability of the cardiomyocytes. Cardiomyocytes in the logarithmic phase were seeded into 96-well plates at a density of $\sim 6 \times 10^{4}$ cells/ml/group, and maintained in a $5 \% \mathrm{CO}_{2}$ atmosphere at $37^{\circ} \mathrm{C}$ for $12 \mathrm{~h}$. Subsequently, the cardiomyocytes were maintained for 12, 24 and $48 \mathrm{~h}$. A total of $10 \mu \mathrm{l} \mathrm{CCK}$ reagent was supplemented into the wells of the 96-well plates. The cardiomyocytes were maintained for $3 \mathrm{~h}$. A microplate reader (Bio-Rad Laboratories, Inc., Hercules, CA, USA) was used to record the absorbance at $450 \mathrm{~nm}$. Cell viability was evaluated as the percentage of cell survival compared with the control.

Enzyme activity detection. The lactate dehydrogenase (LDH; cat. no. C0016) detection kit, malondialdehyde (MDA; cat. no. S0131) detection kit, superoxide dismutase (SOD; cat. no. S0101) assay kit and glutathione peroxidase (GPx; cat. no. S0058) assay kit were purchased from Beyotime Institute of Biotechnology. Detection of MDA content, LDH activity, SOD activity and Gpx activity were performed according to the manufacturer's protocols.

Reactive oxygen species (ROS) levels were detected using 2',7'-dichlorodihydrofluorescein diacetate (DCFH-DA; Sigma-Aldrich; Merck KGaA, Darmstadt, Germany). A total of $2 \times 10^{6}$ cells $/ \mathrm{ml}$ were seeded in a 6 -well plate and $10 \mathrm{mM}$ DCFH-DA in PBS was added to the cells for $20 \mathrm{~min}$ at $37^{\circ} \mathrm{C}$. DCF fluorescence was measured using a BD LSR II flow cytometer (BD Biosciences, Franklin Lakes, NJ, USA). The flow cytometry data were analyzed using FlowJo software version 7.5.5 (FlowJo LLC, Ashland, OR, USA).

Apoptosis assay. Flow cytometry (FCM) was conducted to assess the apoptosis of cardiomyocytes. Following washing with PBS, cultured cardiomyocytes were trypsinized with $0.25 \%$ trypsin (Beyotime Institute of Biotechnology). The cells were harvested and centrifuged at $1,000 \mathrm{x} \mathrm{g}$ for $5 \mathrm{~min}$ at $4^{\circ} \mathrm{C}$. The supernatant was removed and the cardiomyocytes were suspended in the incubation buffer at a density of $1 \times 10^{6}$ cells $/ \mathrm{ml}$ for assessment. Apoptosis of cardiomyocytes was detected with the Annexin V-fluorescein isothiocyanate and propidium iodide detection kit (cat. no. V13242, Invitrogen; Thermo Fisher Scientific, Inc.), according to the manufacturer's instructions. A flow cytometer (FACSCalibur) running BD CellQuest ${ }^{\mathrm{TM}}$ software version 1.2 (both from BD Biosciences) was used to assess cell apoptosis.

Evaluation of $\mathrm{Ca}^{2+}$ and MMP in cardiomyocytes. PBS was added to the cultured cardiomyocytes until the cell concentration reached $1 \times 10^{6}$ cells $/ \mathrm{ml}$. Rho $123(5 \mu \mathrm{M}$; cat. no. R8004; Sigma-Aldrich; Merck KGaA) or Fluo-3AM [3 $\mu \mathrm{M}$; cat. no. 70-F1243; Multisciences (Lianke) Biotech Co., Ltd., Hangzhou, China] were added to the cardiomyocytes. The cardiomyocytes were incubated at room temperature and kept away from light for $10 \mathrm{~min}$. FCM was performed to assess the MMP and $\mathrm{Ca}^{2+}$ level in cardiomyocytes, and the excitation wavelength was $488 \mathrm{~nm}$. In total, 10,000 cells were collected from each sample. The data was analyzed using BD CellQuest ${ }^{\mathrm{TM}}$ software version 1.2 (BD Biosciences).

Evaluation of mitochondrial permeability transition pore $(m P T P)$ in cardiomyocytes. Mitochondria were isolated from cells using a Mitochondrial extraction kit (cat. no. SM0020; Beijing Solarbio Science \& Technology Co., Ltd.), according 
to the manufacturer's protocols. Tumescent fluid (containing $120 \mathrm{mmol} / 1 \mathrm{KCl}, 20 \mathrm{mmol} / 1 \mathrm{MOPS}, 10 \mathrm{mmol} / 1 \mathrm{Tris}-\mathrm{HCl}$ and $5 \mathrm{mmol} / 1 \mathrm{KH}_{2} \mathrm{PO}_{4} ; \mathrm{pH}$ 7.4; Beyotime Institute of Biotechnology) was added to the mitochondria and the mixture was diluted to the point where the protein concentration reached $0.25 \mathrm{~g} / 1$ at $25^{\circ} \mathrm{C}$. Subsequently, $200 \mu \mathrm{mol} / 1 \mathrm{CaCl}_{2}$ was added to the mitochondria and the absorbance at $520 \mathrm{~nm}$ was observed for $15 \mathrm{~min}$. The alterations in absorbance suggested the degree of mitochondrial swelling, which indicated the opening of mPTP.

Western blot analysis. Proteins were isolated using NP-40 lysis buffer (Beyotime Institute of Biotechnology, China). The BCA Protein quantification kit (cat. no. ab207002; Abcam, Cambridge, UK) was used to determine protein concentration. A total of $25 \mu \mathrm{g} /$ lane protein was separated by $12 \%$ SDS-PAGE. The separated products were transferred to a polyvinylidene difluoride membrane (EMD Millipore, Billerica, MA, USA). The membranes were blocked with $5 \%$ skimmed milk at room temperature for $2 \mathrm{~h}$. Western blotting was performed with specific primary antibodies at $4^{\circ} \mathrm{C}$ overnight: anti-inactive-caspase (1:2,000; cat. no. ab184787; rabbit anti-rat); anti-active-caspase-3 (1:500; cat. no. ab49822; rabbit anti-rat); anti-inactive-caspase-9 (1:500; cat. no. ab138412; rabbit anti-rat); anti-active-caspase-9 (1:1,000; cat. no. ab25758; rabbit anti-rat); anti-mitochondrial cyto $c$ (1:5,000; cat. no. ab133504; rabbit anti-rat); anti-mitochondrial AIF (1:1,000; cat. no. ab32516; rabbit anti-rat); anti-cyto $c$ (1:1,000; cat. no. ab110325; rabbit anti-rat); anti-AIF (1:1,000; cat. no. ab1998; rabbit anti-rat); anti-cyclooxygenase (COX) IV (1:2,000; cat. no. ab16056; rabbit anti-rat); and anti-actin (1:5,000; cat. no. ab179467; rabbit anti-rat) (all from Abcam). Horseradish peroxidase-conjugated secondary antibodies (1:5,000; Abcam; cat. no. ab205718; goat anti-rabbit) were supplemented and incubated at room temperature for $1 \mathrm{~h}$. Enhanced chemiluminescent (ECL) reagents (EMD Millipore) in combination with an ECL system (GE Healthcare, Chicago, IL, USA) were used to assess the results. The density of the protein bands was quantified using Quantity One ${ }^{\circledR}$ software version 4.2.1 (Bio-Rad Laboratories, Inc.).

Reverse transcription-quantitative PCR (RT-qPCR) analysis. Total RNA was extracted from the cultured cardiomyocytes using TRIzol ${ }^{\circledR}$ reagent (Thermo Fisher Scientific, Inc.). RNA was reverse transcribed to cDNA using BeyoRT ${ }^{\mathrm{TM}}$ II cDNA Synthesis kit (Beyotime Institute of Biotechnology), according to the manufacturer's protocol. qPCR was performed using a SYBR-Green PCR Master Mix kit (Applied Biosystems; Thermo Fisher Scientific, Inc.) on a ABI 7500 Thermocycler (Applied Biosystems). PCR cycling conditions were as follows: $10 \mathrm{~min}$ pretreatment at $94^{\circ} \mathrm{C}$, at $95^{\circ} \mathrm{C}$ for $15 \mathrm{sec}$, at $68^{\circ} \mathrm{C}$ for $45 \mathrm{sec}\left(45\right.$ cycles), at $95^{\circ} \mathrm{C}$ for $15 \mathrm{sec}$, at $68^{\circ} \mathrm{C}$ for $1 \mathrm{~min}$, at $94^{\circ} \mathrm{C}$ for $15 \mathrm{sec}$, a final extension at $75^{\circ} \mathrm{C}$ for $10 \mathrm{~min}$ and held at $4^{\circ} \mathrm{C}$. The primers were purchased from Invitrogen (Thermo Fisher Scientific, Inc.): Caspase-3, forward, 5'-TGTCGATGCAGC TAACCTCA-3' and reverse, 5'-GCAGTAGTCGCCTCTGAA GA-3' (product: $241 \mathrm{bp}$ ); caspase-9, forward, 5'-CATTGGTTC TGGCAGAGCTC-3' and reverse, 5'-AGCAGTCAGGTCGTT CTTCA-3' (product: $238 \mathrm{bp}$ ); cyto $c$, forward, 5'-CAACTG CACAAGACAACCCA-3' and reverse, 5'-ATAGCACAATCC
CCACCACA-3' (product: 204 bp); AIF, forward, 5'-ACATGC GACCTCCTCTTTCA-3' and reverse, 5'-TGCCTCTTACAT CCAGGTGG-3' (product: 213 bp); COX IV, forward, 5'-TGA AGGAGAAGGAGAAGGCC-3' and reverse, 5'-ACCCAG TCACGATCAAAGGT-3' (product: 229 bp); actin, forward, 5'-CAACATGGATGAGCGGAAGG-3' and reverse, 5'-GCA GTGTAGCAGCATCGAAA-3' (product: 233 bp). Actin was used as the control of the input RNA level. The $2^{-\Delta \Delta \mathrm{Cq}}$ method was used to analyze the relative gene expression (28).

Statistical analysis. Results in the present study are presented as the mean \pm standard error of the mean with at least three independent experiments repeated. All experimental data were analyzed one-way analysis of variance followed by the Dunnett's test. $\mathrm{P}<0.05$ was considered to indicate a statistically significant difference. Data analysis was performed using GraphPad Prism version 6.0 (GraphPad Software, Inc., La Jolla, CA, USA).

\section{Results}

$R P$ increases the cell viability of $M I / R$-induced cardiomyocytes. The cell viabilities of cardiomyocytes treated with different concentrations of RP were measured in the present study. It was observed that compared with the control group, the cell activities of cardiomyocytes treated with RP at concentrations of 10, 20, 40 and $80 \mu \mathrm{M}$ exhibited no significant difference (Fig. 1A). However, it was identified that with the treatments of 160 and $320 \mu \mathrm{M}$ RP, the cell viabilities of cardiomyocytes were significantly decreased, particularly at $48 \mathrm{~h}$ treatment $(\mathrm{P}<0.05$; Fig. $1 \mathrm{~A})$. These results suggested that low concentrations of RP exhibited a limited effect on the cell viability of cardiomyocytes. Therefore, the cell viabilities of cardiomyocytes subjected to MI/R injury and MI/R-induced cardiomyocytes treated with 20,40 and $80 \mu \mathrm{M}$ RP were further evaluated. According to the results, it was observed that MI/R injury decreased the cell viability of cardiomyocytes; whereas, RP increased the cell viability of MI/R-induced cardiomyocytes in a dose-dependent manner (Fig. 1B). The results indicated that RP was able to enhance the cell viability of cardiomyocytes, which were subjected to MI/R injury. Therefore, it was confirmed that MI/R resulted in low cell viability of cardiomyocytes; whereas, RP increased the cell viability of MI/R-induced cardiomyocytes within a certain concentration range.

$R P$ decreases the oxidative stress and mPTP level of $M I / R$-induced cardiomyocytes. Oxidative stress has been proposed to contribute to the development and progression of MI/R (5). Therefore, the levels of oxidative stress markers, including ROS, MDA, LDH, SOD and GPx in cardiomyocytes treated with $\mathrm{MI} / \mathrm{R}$ and different concentrations of RP were assessed. The ROS, MDA and LDH content in cardiomyocytes treated with $\mathrm{MI} / \mathrm{R}$ in advance were markedly higher compared with the control, while treatment with $\geq 40 \mu \mathrm{M}$ RP significantly decreased the ROS, MDA and LDH content in MI/R-induced cardiomyocytes $(\mathrm{P}<0.05$; Fig. 2A-C). However, MI/R was observed to be able to reduce the activities of SOD and GPX in cardiomyocytes. The SOD and GPx activity in MI/R-induced cardiomyocytes was significantly increased $(\mathrm{P}<0.05 ;$ Fig. $2 \mathrm{D}$ 

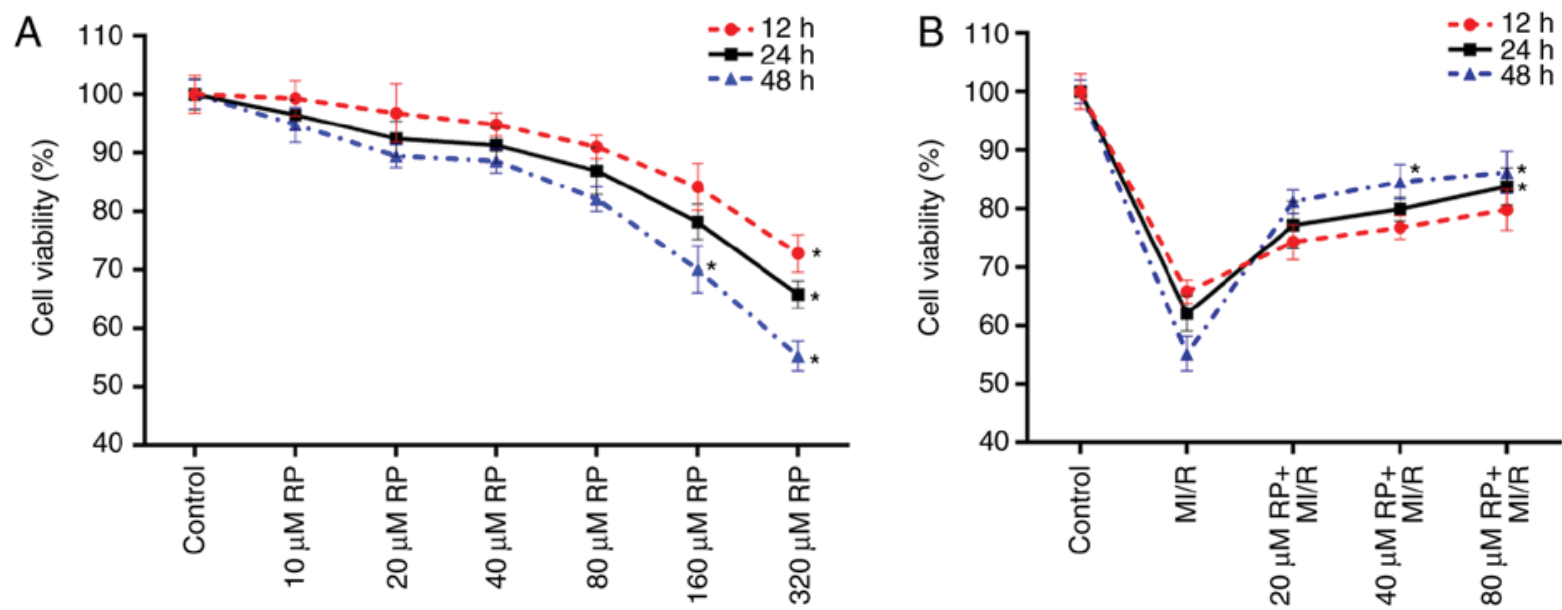

Figure 1. RP increases the cell viability of MI/R-induced cardiomyocytes. (A) CCK-8 assay was performed on the cell viability of cardiomyocytes treated with different concentrations of RP. (B) CCK-8 assay was conducted to test the cell viability of cardiomyocytes treated with MI/R, $20 \mu \mathrm{M} \mathrm{RP}+\mathrm{MI} / \mathrm{R}, 40 \mu \mathrm{M}$ $\mathrm{RP}+\mathrm{MI} / \mathrm{R}$ and $80 \mu \mathrm{M} \mathrm{RP}+\mathrm{MI} / \mathrm{R}$, respectively. ${ }^{*} \mathrm{P}<0.05$ vs. Control. RP, rhynchophylline; MI/R, myocardial ischemia-reperfusion; CCK-8, Cell Counting Kit-8.
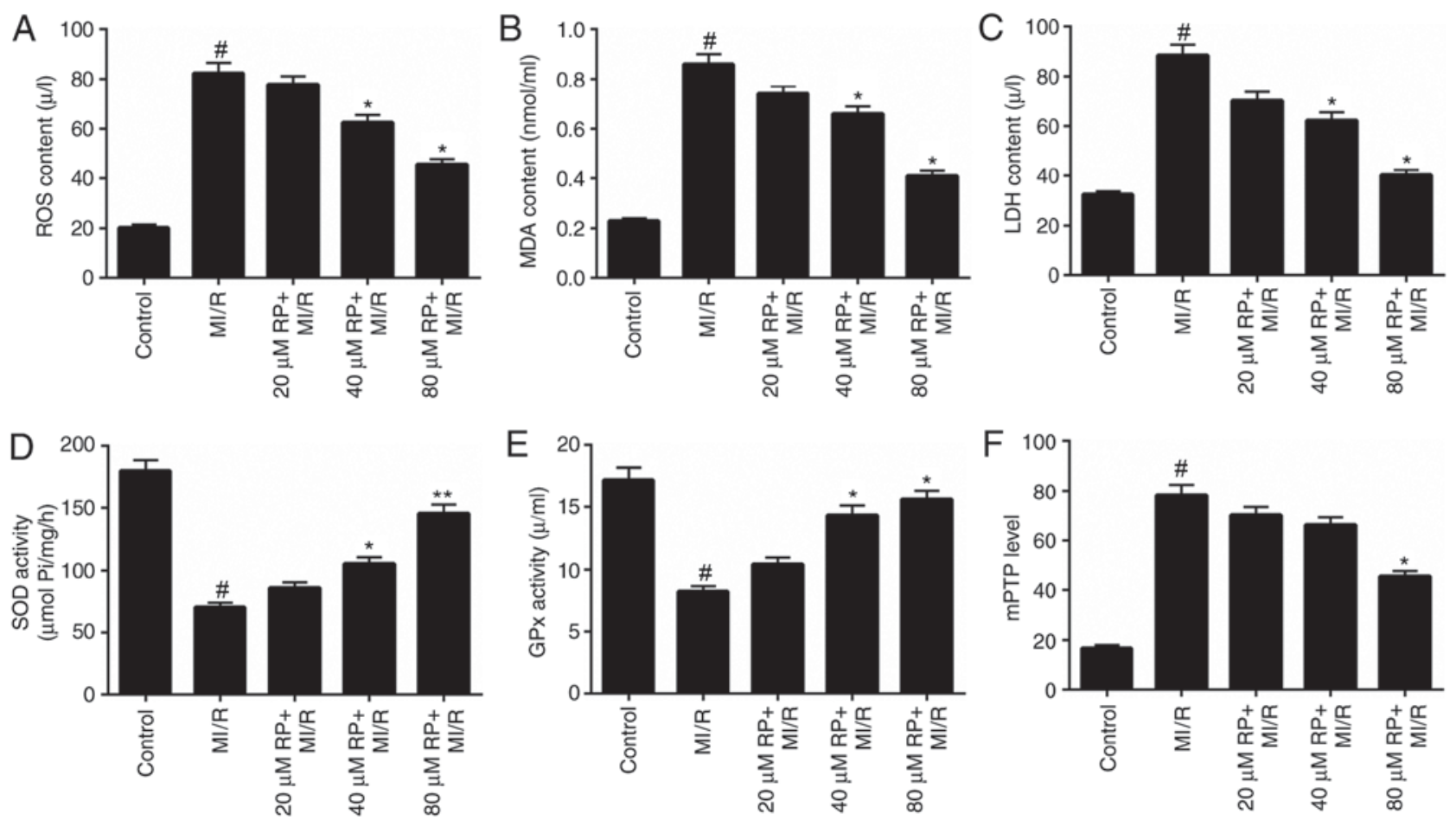

Figure 2. RP decreases the oxidative stress and mPTP level of MI/R-induced cardiomyocytes. Cardiomyocytes were treated with MI/R, $20 \mu \mathrm{M} \mathrm{RP}+\mathrm{MI} / \mathrm{R}, 40 \mu \mathrm{M}$ $\mathrm{RP}+\mathrm{MI} / \mathrm{R}$ and $80 \mu \mathrm{M} \mathrm{RP}+\mathrm{MI} / \mathrm{R}$. (A) ROS levels were detected by flow cytometric analysis. (B) MDA content, (C) LDH activity, (D) SOD activity and (E) GPx activity in cardiomyocytes were measured using commercial kits. (F) Spectrophotometry was performed to detect the degree of mPTP openness in cardiomyocytes. ${ }^{\sharp} \mathrm{P}<0.05$ vs. Control; ${ }^{*} \mathrm{P}<0.05,{ }^{* * *} \mathrm{P}<0.01$ vs. MI/R. RP, rhynchophylline; mPTP, mitochondrial permeability transition pore; ROS, reactive oxygen species; MDA, malondialdehyde; LDH, lactate dehydrogenase; SOD, superoxide dismutase; GPx, glutathione peroxidase; MI/R, myocardial ischemia-reperfusion.

and E) following treatment with $\geq 40 \mu \mathrm{M}$ RP. It was suggested that RP reduced oxidative stress in MI/R-induced cardiomyocytes. The opening degree of mPTP in cardiomyocytes was assessed by spectrophotometry. The results revealed that $\mathrm{MI} / \mathrm{R}$ increased the $\mathrm{mPTP}$ opening degree in cardiomyocytes $(\mathrm{P}<0.05$; Fig. 2F); whereas, RP decreased the opening degree of mPTP in MI/R-induced cardiomyocytes.

$R P$ modulates the $\mathrm{Ca}^{2+}$ and MMP levels in MI/R-induced cardiomyocytes. Furthermore, the $\mathrm{Ca}^{2+}$ and MMP levels in cardiomyocytes treated with $\mathrm{MI} / \mathrm{R}$ and different concentrations of RP were measured. Based on the FCM data, it was identified that MI/R markedly increased the $\mathrm{Ca}^{2+}$ level in cardiomyocytes. Following treatment with 40 and $80 \mu \mathrm{M} \mathrm{RP}$, the $\mathrm{Ca}^{2+}$ level in MI/R-induced cardiomyocytes was significantly decreased compared with the MI/R group ( $\mathrm{P}<0.05$; Fig. $3 \mathrm{~A})$, suggesting that RP was able to decrease the $\mathrm{Ca}^{2+}$ level in MI/R-induced cardiomyocytes. Additionally, the FCM results on MMP revealed that MI/R significantly decreased the MMP level in cardiomyocytes; whereas, treatment with 40 and $80 \mu \mathrm{M}$ RP 

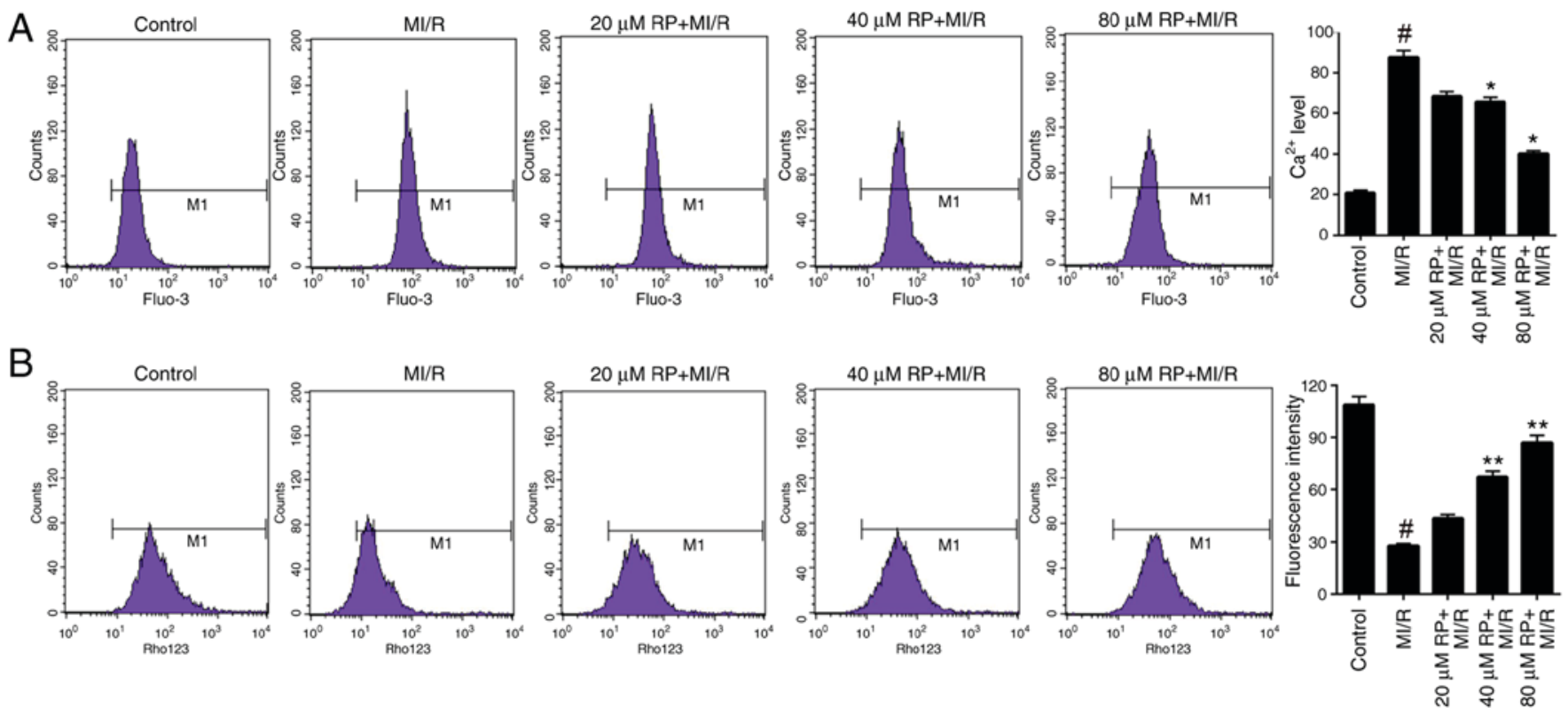

Figure 3. RP modulates the (A) $\mathrm{Ca}^{2+}$ and (B) MMP levels in MI/R-induced cardiomyocytes. Flow cytometry was performed to assess the Ca ${ }^{2+}$ and $\mathrm{MMP}$ levels of cardiomyocytes treated, with $\mathrm{MI} / \mathrm{R}, 20 \mu \mathrm{M} \mathrm{RP}+\mathrm{MI} / \mathrm{R}, 40 \mu \mathrm{M} \mathrm{RP}+\mathrm{MI} / \mathrm{R}$ and $80 \mu \mathrm{M} \mathrm{RP}+\mathrm{MI} / \mathrm{R}$. ${ }^{*} \mathrm{P}<0.05$ vs. Control; ${ }^{*} \mathrm{P}<0.05$, ${ }^{* *} \mathrm{P}<0.01 \mathrm{vs}$. MI/R. $\mathrm{Rp}$, rhynchophylline; MMP, mitochondrial membrane potential; MI/R, myocardial ischemia-reperfusion.

was observed to significantly increase the MMP levels in MI/R-induced cardiomyocytes $(\mathrm{P}<0.01$; Fig. 3B). These results suggested that RP was able to modulate the $\mathrm{Ca}^{2+}$ and MMP levels in MI/R-induced cardiomyocytes.

$R P$ regulates mitochondrial-associated gene expression. The mitochondrial mechanisms of RP in protecting cardiomyocytes against $\mathrm{MI} / \mathrm{R}$ induced injury were examined. In this analysis, the mitochondrial cyto $c$, mitochondrial AIF, cytosolic cyto $c$ and cytosolic AIF expression in cardiomyocytes were measured. In the cardiomyocytes from the MI/R groups, the mitochondrial cyto $c$ expression was decreased compared with the control, while the expression level of mitochondrial AIF was markedly increased compared with the control. Increases were observed in the mitochondrial cyto $c$ expression in $\mathrm{MI} / \mathrm{R}$-induced cardiomyocytes treated with 40 and $80 \mu \mathrm{M} \mathrm{RP}$. It was additionally identified that RP was able to downregulate the expression level of mitochondrial AIF in MI/R-induced cardiomyocytes, with significant decreases observed in the 40 and $80 \mu \mathrm{M} \mathrm{RP}+\mathrm{MI} / \mathrm{R}$ groups $(\mathrm{P}<0.01$; Fig. $4 \mathrm{~A}$ and $\mathrm{B})$. Additionally, the expression level of cytosolic cyto $c$ in cardiomyocytes was upregulated upon MI/R injury, while cytosol AIF expression was reduced by MI/R injury $(\mathrm{P}<0.05)$. Following treatment with RP, the expression level of cytosolic cyto $c$ was decreased; whereas, the cytosolic AIF expression was significantly increased in MI/R-induced cardiomyocytes $(\mathrm{P}<0.05$; Fig. 4C and D). Therefore, it was demonstrated that RP upregulated the mitochondrial cyto $c$ and cytosolic AIF expression, and downregulated the expression levels of mitochondrial AIF and cytosolic cyto $c$ in MI/R-induced cardiomyocytes.

$R P$ suppresses the MI/R-induced apoptosis of cardiomyocytes. From the aforementioned results, it was determined that $\mathrm{RP}$ was able to enhance the cell viability of MI/R-induced cardiomyocytes; therefore, the apoptotic capacity of $\mathrm{MI} / \mathrm{R}$-induced cardiomyocytes treated with RP was evaluated. It was demonstrated that the proportion of apoptotic cardiomyocytes in MI/R group was $26.46 \%$, which was significantly increased compared with the control (6.32\%). Furthermore, following treatment with 20, 40 and $80 \mu \mathrm{M}$ RP, the percentage of apoptotic MI/R-induced cardiomyocytes decreased from 26.46 to $23,18.52$ and $14.1 \%$, respectively (Fig. 5). In particular, significant decreases in the percentage of apoptotic cells were observed in the 40 and $80 \mu \mathrm{M} \mathrm{RP}+\mathrm{MI} / \mathrm{R}$ groups $(\mathrm{P}<0.05)$. These data suggested that RP markedly decreased the apoptotic capacity of MI/R-induced cardiomyocytes in a dose-dependent manner.

$R P$ affects the apoptosis-associated protein expression. According to the apoptosis results above, it was demonstrated that RP inhibited the apoptosis of cardiomyocytes induced by $\mathrm{MI} / \mathrm{R}$. Therefore, the apoptosis-associated proteins in cardiomyocytes were further investigated. According to the RT-qPCR data, it was observed that the caspase-3 and caspase-9 expression in cardiomyocytes from MI/R group was significantly increased compared with the control; whereas, the caspase-3 and caspase- 9 expressions in MI/R-induced cardiomyocytes were decreased by RP in a dose-dependent manner, with significant differences observed with 40 and $80 \mu \mathrm{M}$ RP $(\mathrm{P}<0.05$; Fig. 6A). Furthermore, the western blot analysis revealed that the expression levels of inactive caspase-3 and -9 in cardiomyocytes were markedly downregulated with $\mathrm{MI} / \mathrm{R}$ injury; however, were upregulated upon treatment with different concentrations of RP. Additionally, it was identified that MI/R upregulated the active caspase-3 and -9 expression in cardiomyocytes. However, RP was able to decrease the expression levels of caspase-3 and -9 in MI/R-induced cardiomyocytes compared with the control (Fig. 6B). Therefore, it was demonstrated that RP modulated 

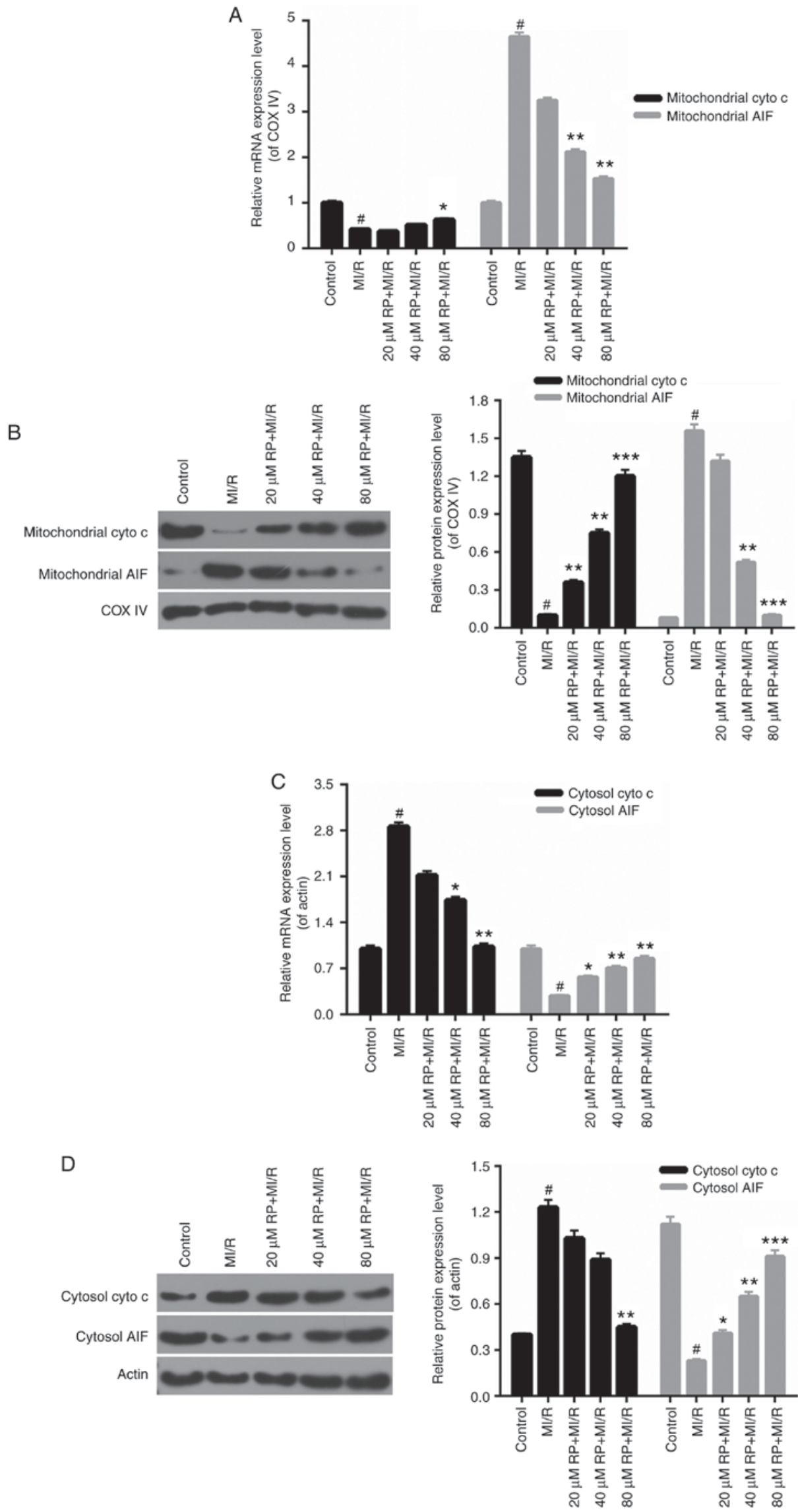

Figure 4. RP regulates the mitochondrial-associated gene expression. Cardiomyocytes were treated with MI/R, $20 \mu \mathrm{M} \mathrm{RP}+\mathrm{MI} / \mathrm{R}, 40 \mu \mathrm{M}$ RP+MI/R and $80 \mu \mathrm{M} \mathrm{RP}+\mathrm{MI} / \mathrm{R}$. (A) RT-qPCR and (B) western blot assays were performed on the expression levels of mitochondrial cyto $c$ and mitochondrial AIF in cardiomyocytes. (C) RT-qPCR and (D) western blot assays were conducted to determine the expression levels of cytosolic cyto $c$ and cytosolic AIF in cardiomyocytes. ${ }^{*} \mathrm{P}<0.05$ vs. Control; ${ }^{*} \mathrm{P}<0.05,{ }^{* *} \mathrm{P}<0.01,{ }^{* * * *} \mathrm{P}<0.001$ vs. MI/R. Rp, rhynchophylline; MI/R, myocardial ischemia-reperfusion; RT-qPCR, reverse transcription-quantitative polymerase chain reaction; cyto $c$, cytochrome $c$; AIF, apoptosis-inducing factor; COX, cyclooxygenase. 

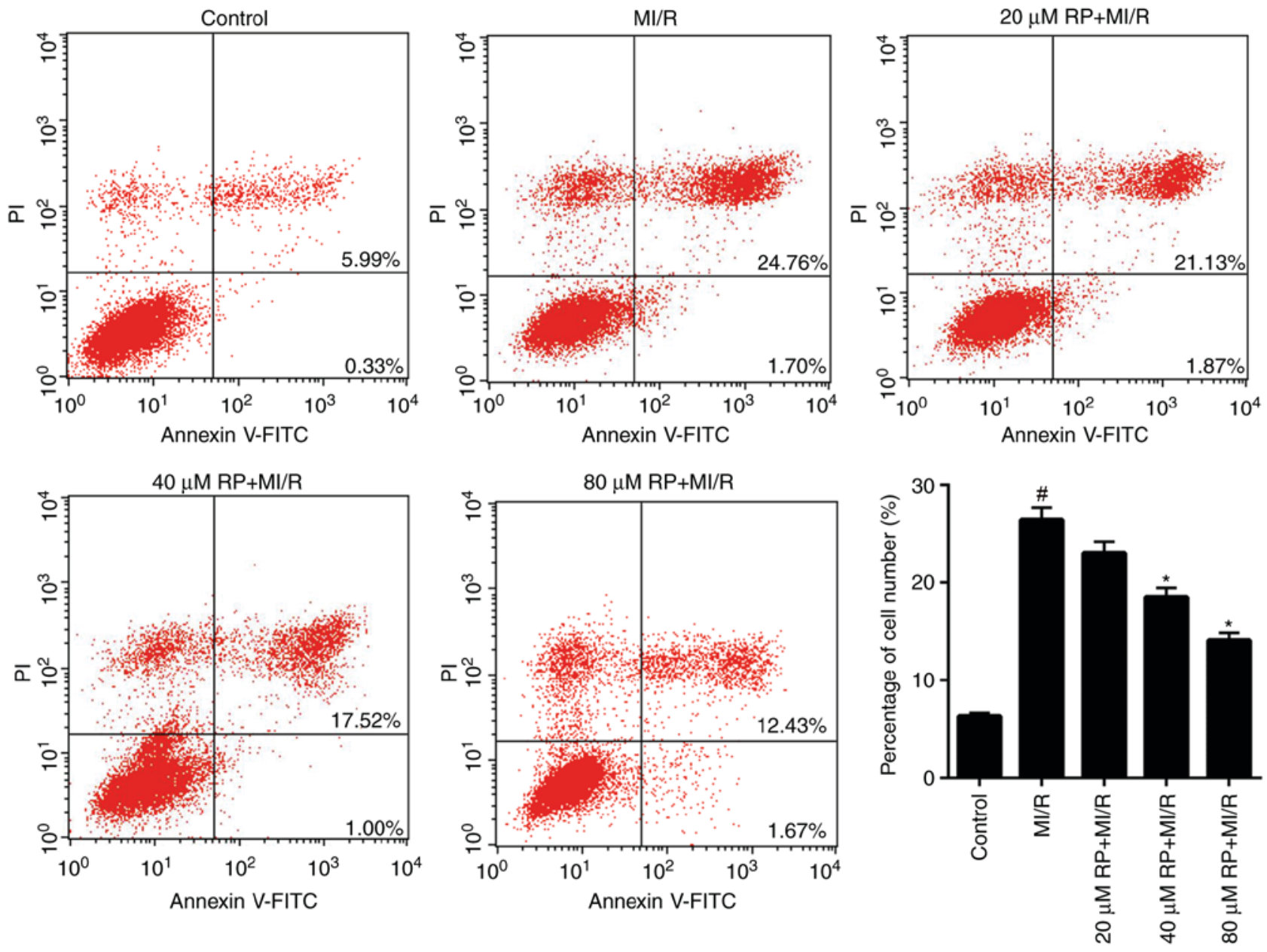

Figure 5. RP suppresses the MI/R-induced apoptosis of cardiomyocytes. Flow cytometry was performed to assess the cell apoptosis of cardiomyocytes, treated with MI/R, $20 \mu \mathrm{M} \mathrm{RP}+\mathrm{MI} / \mathrm{R}, 40 \mu \mathrm{M} \mathrm{RP}+\mathrm{MI} / \mathrm{R}$ and $80 \mu \mathrm{M} \mathrm{RP}+\mathrm{MI} / \mathrm{R}$. "P<0.05 vs. Control; "P<0.05 vs. MI/R. Rp, rhynchophylline; MI/R, myocardial ischemia-reperfusion; PI, propidium iodide; FITC, fluorescein isothiocyanate.

the apoptosis-associated protein expression in MI/R-induced cardiomyocytes.

\section{Discussion}

Cardiomyocyte injury is a critical cytological basis for stress-induced cardiovascular disease. Cell apoptosis, a crucial cytological mechanism of cardiomyocyte injury, serves a role in a number of cardiovascular diseases, including heart failure, AMI, atherosclerosis and MI/R injury (29). RP represents a crucial active substance isolated from Chinese herbal medicine. Previous studies demonstrated that RP had neuroprotective functions (30,31). Additionally, Cao et al (32) suggested that RP may prevent cardiac dysfunction and improve survival in lipopolysaccharide-challenged mice by suppressing macrophage inhibitor- $\kappa \mathrm{B} \alpha$ phosphorylation. However, the roles of $\mathrm{RP}$ in MI/R injury remain unclear. Therefore, an aim of the present study was to investigate the effect of RP in the MI/R injury and the associated mechanisms. The cell viabilities of cardiomyocytes treated with different concentrations of RP were measured to assess the cytotoxicity of RP. It was identified that the cell viability of cardiomyocytes began to decrease after the cells were treated with $160 \mu \mathrm{M} \mathrm{RP}$ for $48 \mathrm{~h}$. Therefore, the cell viability of cardiomyocytes treated with MI/R injury in addition to using 20, 40 and $80 \mu \mathrm{M}$ RP, was further assessed. The results demonstrated that MI/R injury inhibited the cell viability of cardiomyocytes, while RP markedly increased the cell viability of MI/R-induced cardiomyocytes, particularly with treatment duration of $48 \mathrm{~h}$. Therefore, it was demonstrated that RP increased the cell viability of cardiomyocytes subjected to MI/R injury.

It was demonstrated that acute stress may lead to spasmodic cardiac arrhythmia and sudden mortality (33). Long-term high-intensity oxidative stress additionally causes a variety of serious cardiovascular diseases, for instance, hypertension and atherosclerosis $(10,34)$. Additionally, it has been identified that MI/R injury stimulates severe oxidative stress in cardiomyocytes (35-37). Therefore, to identify whether RP was able to modulate the oxidative stress in cardiomyocytes subjected to MI/R injury, the levels of oxidative stress markers in cardiomyocytes were assessed. From the present results, it was observed that RP markedly reduced the levels of ROS, MDA and LDH, and increased the SOD and GPx activities in MI/R-induced cardiomyocytes. These results suggested that RP may function to reduce oxidative stress in the cardiomyocytes with MI/R injury. Therefore, it was concluded that RP is able to reduce the oxidative stress in cardiomyocytes subjected to $\mathrm{MI} / \mathrm{R}$ injury. 

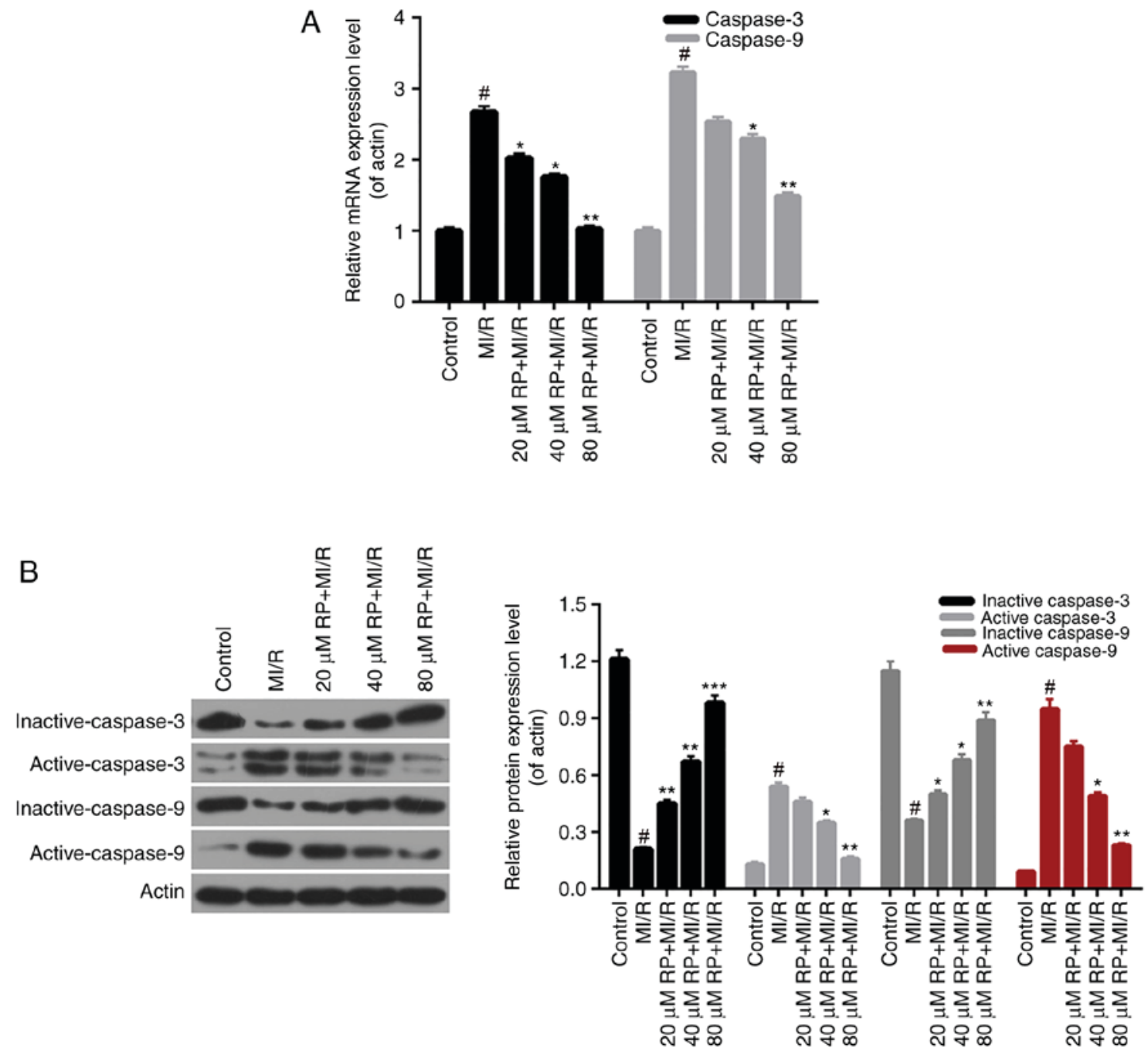

Figure 6. RP affects the apoptosis-associated protein expression. (A) Reverse transcription-quantitative polymerase chain reaction and (B) western blotting assays were conducted to evaluate the expression levels of inactive-caspase-3, active-caspase-3, inactive-caspase- 9 and active-caspase- 9 in cardiomyocytes treated, respectively, with $\mathrm{MI} / \mathrm{R}, 20 \mu \mathrm{M} \mathrm{RP}+\mathrm{MI} / \mathrm{R}, 40 \mu \mathrm{M} \mathrm{RP}+\mathrm{MI} / \mathrm{R}$ and $80 \mu \mathrm{M} \mathrm{RP}+\mathrm{MI} / \mathrm{R} .{ }^{*} \mathrm{P}<0.05$ vs. Control; ${ }^{*} \mathrm{P}<0.05,{ }^{* *} \mathrm{P}<0.01$ and ${ }^{* * * *} \mathrm{P}<0.001$ vs. $\mathrm{MI} / \mathrm{R}$. $\mathrm{Rp}$, rhynchophylline; MI/R, myocardial ischemia-reperfusion.

Previous studies suggested that mitochondria serve as an important pathway of stress-induced cardiomyocyte injury $(38,39)$. Mitochondria not only provide ATP to cells but additionally participate in multiple cytopathic processes $(40,41)$. Mitochondria act as important pathways that mediate oxidative stress and cell damage in organisms. Previous studies demonstrated that the mPTP opening degree, $\mathrm{Ca}^{2+}$ disturbances and MMP levels serve crucial roles in the modulation of oxidative stress (42-44). Therefore, the mPTP, $\mathrm{Ca}^{2+}$ and MMP levels were evaluated in cardiomyocytes with $\mathrm{MI} / \mathrm{R}$ injury and treated with different concentrations of RP to investigate the effect of RP on the mitochondria. Based on the present results, it was observed that RP reduced the opening degree of $\mathrm{mPTP}$ in MI/R-induced cardiomyocytes. The FCM data revealed that RP decreased the $\mathrm{Ca}^{2+}$ level; however, it increased the MMP level in MI/R-induced cardiomyocytes. These results suggested that RP was able to affect the functions of mitochondria in the cardiomyocytes with $\mathrm{MI} / \mathrm{R}$ injury. It was demonstrated that an increased MMP level may result in the release of mitochondrial cyto $c$, AIF, Smac and other apoptosis-inducing factor, resulting in cell apoptosis (17-19). In order to further investigate the accurate mechanisms of RP in the modulation of mitochondria function, the associated mitochondrial-associated mechanisms in cardiomyocytes were assessed. It was identified that in mitochondria of MI/R-induced cardiomyocytes, RP markedly upregulated cyto $c$ expression, while it decreased the expression level of AIF. Nevertheless, the cyto $c$ and AIF expression modulations in the cytosol of MI/R-induced cardiomyocytes by RP were reversed to the expression of those in mitochondria. RP markedly downregulated the cyto $c$ expression, while it upregulated the AIF expression in the cytosol of MI/R-induced cardiomyocytes. Therefore, it was hypothesized that RP is able to reduce the release of cyto $c$ from the mitochondria into cytosol and stimulate the release of AIF from mitochondria into the cytosol. Collectively, it was demonstrated that RP affected the functions of mitochondria by regulating the mitochondrial mechanisms in cardiomyocytes suffering from MI/R injury.

Sinha et al (45) suggested that oxidative stress may cause cellular apoptosis via the mitochondria-dependent pathway. Based on the results described above, it was demonstrated that 
RP affected the oxidative stress and mitochondria mechanisms in cardiomyocytes suffering from MI/R injury. Therefore, it was hypothesized that RP may affect the apoptotic capacity of cardiomyocytes induced by MI/R injury. Therefore, the cell apoptosis of cardiomyocytes treated with MI/R injury at 20,40 and $80 \mu \mathrm{M}$ of RP was evaluated. The FCM data indicated that MI/R markedly accelerated the apoptosis ability of cardiomyocytes, while RP evidently suppressed the apoptosis of MI/R-induced cardiomyocytes in a dose-dependent manner. These results demonstrated that RP was able to suppress the apoptosis of cardiomyocytes induced by MI/R injury. In order to further examine the accurate mechanisms of RP underlying the protection of cardiomyocytes against MI/R injury, the apoptosis-associated mechanisms were investigated. The RT-qPCR and western blotting data confirmed that RP markedly downregulated the expression levels of caspase-3 and caspase-9 in MI/R-induced cardiomyocytes. Therefore, it was demonstrated that RP suppressed the apoptosis of $\mathrm{MI} / \mathrm{R}$-induced cardiomyocytes by regulating the expression levels of caspase-3 and caspase-9.

In conclusion, the present study demonstrated that RP ameliorated MI/R injury through the modulation of mitochondrial mechanisms. The present results have provided novel insight into the mechanisms of RP and cardiomyocytes. The potential effects of RP on the protection of MI/R-induced cardiomyocytes suggest that RP may be an effective target for $\mathrm{MI} / \mathrm{R}$ injury therapy.

\section{Acknowledgements}

Not applicable.

\section{Funding}

No funding was received.

\section{Availability of data and materials}

All data generated and/or analyzed during this study are included in this published article.

\section{Authors' contributions}

QJQ, LQC and MLG designed the study. QJQ, LQC, PL, YBW and XZZ performed the experiments. PL, YBW and XZZ performed data analysis. QJQ wrote the main manuscript. QJQ, LQC, PL and MLG contributed to manuscript revisions. All authors reviewed the manuscript.

\section{Ethics approval and consent to participate}

Not applicable.

\section{Patient consent for publication}

Not applicable.

\section{Competing interests}

The authors declare that they have no competing interests.

\section{References}

1. McCullough PA: Coronary artery disease. Clin J Am Soc Nephrol 2: 611-616, 2007.

2. Roger VL, Go AS, Lloyd-Jones DM, Adams RJ, Berry JD, Brown TM, Carnethon MR, Dai S, De Simone G, Ford ES, et al: Heart disease and stroke statistics-2011 update: A report from the american heart association. Circulation 123: e18-e209, 2011.

3. GBD 2013 Mortality and Causes of Death Collaborators: Global, regional, and national age-sex specific all-cause and cause-specific mortality for 240 causes of death, 1990-2013: A systematic analysis for the Global Burden of Disease Study 2013. Lancet 385: 117-171, 2015.

4. Rodriguez-Sinovas A, Abdallah Y, Piper HM and GarciaDorado D: Reperfusion injury as a therapeutic challenge in patients with acute myocardial infarction. Heart Fail Rev 12: 207-216, 2007.

5. Bolli R: Preconditioning: A paradigm shift in the biology of myocardial ischemia. Am J Physiol Heart Circ Physiol 292: H19-H27, 2007.

6. Kitakaze M: How to mediate cardioprotection in ischemic hearts-accumulated evidence of basic research should translate to clinical medicine. Cardiovasc Drugs Ther 24: 217-223, 2010.

7. Tissier R, Waintraub X, Couvreur N, Gervais M, Bruneval P, Mandet C, Zini R, Enriquez B, Berdeaux A and Ghaleh B: Pharmacological postconditioning with the phytoestrogen genistein. J Mol Cell Cardiol 42: 79-87, 2007.

8. Chida Y and Hamer M: Chronic psychosocial factors and acute physiological responses to laboratory-induced stress in healthy populations: A quantitative review of 30 years of investigations. Psychol Bull 134: 829-885, 2008.

9. Sinha R: Chronic stress, drug use, and vulnerability to addiction. Ann N Y Acad Sci 1141: 105-130, 2008.

10. Ma XL, Kumar S, Gao F, Louden CS, Lopez BL, Christopher TA, Wang C, Lee JC, Feuerstein GZ and Yue TL: Inhibition of p38 mitogen-activated protein kinase decreases cardiomyocyte apoptosis and improves cardiac function after myocardial ischemia and reperfusion. Circulation 99: 1685-1691, 1999.

11. Schwartz AR, Gerin W, Davidson KW, Pickering TG, Brosschot JF, Thayer JF, Christenfeld N and Linden W: Toward a causal model of cardiovascular responses to stress and the development of cardiovascular disease. Psychosom Med 65: 22-35, 2003.

12. Bozner A, Balaz V, Dostal J and Szigetiova A: Electron microscopy morphometric analysis of the effect of immobilization stress on the structure of the myocardium in rats belonging to 2 age groups. Cesk Patol 20: 146-150, 1984 (In Slovak).

13. Jonsson L, Johansson G, Lannek N, Lindberg P and Poupa O: Histochemical and electron microscopic studies of acute cardiomyopathy induced by restraint stress in pigs. Recent Adv Stud Cardiac Struct Metab 6: 461-470, 1975.

14. Jonsson L and Johansson G: Cardiac muscle cell damage induced by restraint stress. Virchows Arch B Cell Pathol 17: 1-12, 1974.

15. Kuder T: Electron microscopic studies of neurocytes of the pterygopalatine ganglion in the rat after immobilization. Arch Vet Pol 32: 93-102, 1992.

16. Cai Q, Huang S, Zhu Z, Li H, Li Q, Jia N and Liu J: The effects of prenatal stress on expression of p38 MAPK in offspring hippocampus. Int J Dev Neurosci 26: 535-540, 2008.

17. Grinberg M, Sarig R, Zaltsman Y, Frumkin D, Grammatikakis N, Reuveny E and Gross A: tBID Homooligomerizes in the mitochondrial membrane to induce apoptosis. J Biol Chem 277: 12237-12245, 2002.

18. Komarov AP, Rokhlin OW, Yu CA and Gudkov AV: Functional genetic screening reveals the role of mitochondrial cytochrome $\mathrm{b}$ as a mediator of FAS-induced apoptosis. Proc Natl Acad Sci USA 105: 14453-14458, 2008.

19. Perchellet EM, Wang Y, Weber RL, Sperfslage BJ, Lou K, Crossland J, Hua DH and Perchellet JP: Synthetic 1,4-anthracenedione analogs induce cytochrome $c$ release, caspase-9, -3 , and -8 activities, poly(ADP-ribose) polymerase- 1 cleavage and internucleosomal DNA fragmentation in HL-60 cells by a mechanism which involves caspase-2 activation but not Fas signaling. Biochem Pharmacol 67: 523-537, 2004.

20. Ndagijimana A, Wang X, Pan G, Zhang F, Feng H and Olaleye O: A review on indole alkaloids isolated from Uncaria rhynchophylla and their pharmacological studies. Fitoterapia 86: 35-47, 2013. 
21. Kang TH, Murakami Y, Takayama H, Kitajima M, Aimi N, Watanabe $\mathrm{H}$ and Matsumoto K: Protective effect of rhynchophylline and isorhynchophylline on in vitro ischemia-induced neuronal damage in the hippocampus: Putative neurotransmitter receptors involved in their action. Life Sci 76: 331-343, 2004

22. Zhou J and Zhou S: Antihypertensive and neuroprotective activities of rhynchophylline: The role of rhynchophylline in neurotransmission and ion channel activity. JEthnopharmacol 132: $15-27,2010$.

23. Zhang F, Sun AS, Yu LM, Wu Q and Gong QH: Effects of isorhynchophylline on angiotensin II-induced proliferation in rat vascular smooth muscle cells. J Pharm Pharmacol 60: 1673-1678, 2008.

24. He N, Sun A, Wu Q, Huang X and Shi J: Inhibitory effect of rhynchophylline on cardiomyocyte hypertrophy induced by angiotensin II. Chin J Pharmacol Toxicol 24: 255-260, 2010.

25. Doggrell SA and Brown L: Rat models of hypertension, cardiac hypertrophy and failure. Cardiovasc Res 39: 89-105, 1998.

26. Haugen E, Chen J, Wikström J, Grönros J, Gan LM and Fu LX Parallel gene expressions of IL-6 and BNP during cardiac hypertrophy complicated with diastolic dysfunction in spontaneously hypertensive rats. Int J Cardiol 115: 24-28, 2007.

27. Zhou JY and Zhou SW: Isorhynchophylline: A plant alkaloid with therapeutic potential for cardiovascular and central nervous system diseases. Fitoterapia 83: 617-626, 2012.

28. Livak KJ and Schmittgen TD: Analysis of relative gene expression data using real-time quantitative PCR and the 2(-Delta Delta C(T)) methods. Methods 25: 402-408, 2001.

29. Feuerstein GZ and Young PR: Apoptosis in cardiac diseases: Stress- and mitogen-activated signaling pathways. Cardiovasc Res 45: 560-569, 2000.

30. Huang H, Zhong R, Xia Z, Song J and Feng L: Neuroprotective effects of rhynchophylline against ischemic brain injury via regulation of the Akt/mTOR and TLRs signaling pathways. Molecules 19: 11196-11210, 2014.

31. Xu DD, Hoeven R, Rong R and Cho WC: Rhynchophylline protects cultured rat neurons against methamphetamine cytotoxicity. Evid Based Complement Alternat Med 2012: 636091, 2012.

32. Cao W, Wang Y, Lv X, Yu X, Li X, Li H, Wang Y, Lu D, Qi R and Wang H: Rhynchophylline prevents cardiac dysfunction and improves survival in lipopolysaccharide-challenged mice via suppressing macrophage I- $\kappa \mathrm{B} \alpha$ phosphorylation. Int Immunopharmacol 14: 243-251, 2012.

33. Ziegelstein RC: Acute emotional stress and cardiac arrhythmias. JAMA 298: 324-329, 2007

34. Esch T, Stefano GB, Fricchione GL and Benson H: Stress-related diseases-a potential role for nitric oxide. Med Sci Monit 8: RA103-RA118, 2002
35. Frentzou GA, Drinkhill MJ, Turner NA, Ball SG and Ainscough JF: A state of reversible compensated ventricular dysfunction precedes pathological remodelling in response to cardiomyocyte-specific activity of angiotensin II type-1 receptor in mice. Dis Model Mech 8: 783-794, 2015.

36. Ji L, Fu F, Zhang L, Liu W, Cai X, Zhang L, Zheng Q, Zhang H and Gao F: Insulin attenuates myocardial ischemia/reperfusion injury via reducing oxidative/nitrative stress. Am J Physiol Endocrinol Metab 298: E871-E880, 2010

37. Tao L, Gao E, Jiao X, Yuan Y, Li S, Christopher TA, Lopez BL, Koch W, Chan L, Goldstein BJ and Ma XL: Adiponectin cardioprotection after myocardial ischemia/reperfusion involves the reduction of oxidative/nitrative stress. Circulation 115: 1408-1416, 2007.

38. Qian L, Song X, Ren H, Gong J and Cheng S: Mitochondrial mechanism of heat stress-induced injury in rat cardiomyocyte. Cell Stress Chaperones 9: 281-293, 2004.

39. Xinxing W, Hong F, Rui Z, Yun Z, Jingbo G and Lingiia Q: Phosphorylated nerve growth factor-induced clone B (NGFI-B) translocates from the nucleus to mitochondria of stressed rat cardiomyocytes and induces apoptosis. Stress 15: 545-553, 2012.

40. Loeffler M and Kroemer G: The mitochondrion in cell death control: Certainties and incognita. Exp Cell Res 256: 19-26, 2000.

41. Lopez MF, Kristal BS, Chernokalskaya E, Lazarev A, Shestopalov AI, Bogdanova A and Robinson M: High-throughput profiling of the mitochondrial proteome using affinity fractionation and automation. Electrophoresis 21: 3427-3440, 2000.

42. Kim RH, Smith PD, Aleyasin H, Hayley S, Mount MP, Pownall S, Wakeham A, You-Ten AJ, Kalia SK, Horne P, et al: Hypersensitivity of DJ-1-deficient mice to 1-methyl-4-phenyl1,2,3,6-tetrahydropyrindine (MPTP) and oxidative stress. Proc Natl Acad Sci USA 102: 5215-5220, 2005.

43. Liu H, Bowes RC, III, van de Water B, Sillence C, Nagelkerke JF and Stevens JL: Endoplasmic reticulum chaperones GRP78 and calreticulin prevent oxidative stress, $\mathrm{Ca} 2+$ disturbances, and cell death in renal epithelial cells. J Biol Chem 272: 21751-21759, 1997.

44. Satoh T, Enokido Y, Aoshima H, Uchiyama Y and Hatanaka H: Changes in mitochondrial membrane potential during oxidative stress-induced apoptosis in PC12 cells. J Neurosci Res 50: 413-420, 1997.

45. Sinha K, Das J, Pal PB and Sil PC: Oxidative stress: The mitochondria-dependent and mitochondria-independent pathways of apoptosis. Arch Toxicol 87: 1157-1180, 2013. International (CC BY-NC-ND 4.0) License. 